\title{
DARBOUX TRANSFORMATION FOR CLASSICAL ACOUSTIC SPECTRAL PROBLEM
}

\author{
A. A. YUROVA, A. V. YUROV, and M. RUDNEV
}

Received 3 January 2002

\begin{abstract}
We study discrete isospectral symmetries for the classical acoustic spectral problem in spatial dimensions one and two by developing a Darboux (Moutard) transformation formalism for this problem. The procedure follows steps similar to those for the Schrödinger operator. However, there is no one-to-one correspondence between the two problems. The technique developed enables one to construct new families of integrable potentials for the acoustic problem, in addition to those already known. The acoustic problem produces a nonlinear Harry Dym PDE. Using the technique, we reproduce a pair of simple soliton solutions of this equation. These solutions are further used to construct a new positon solution for this PDE. Furthermore, using the dressing-chain approach, we build a modified Harry Dym equation together with its LA pair. As an application, we construct some singular and nonsingular integrable potentials (dielectric permitivity) for the Maxwell equations in a 2D inhomogeneous medium.
\end{abstract}

2000 Mathematics Subject Classification: 35Q51, 35Q53, 35Q55, 35Q58, 35Q60, 37K10, 37K35, 37K40.

1. Introduction. This paper develops the Darboux transformation and dressing-chain formalism for the classical acoustic spectral problem (referred to below just as the "acoustic problem") and the related Harry Dym (HD) equation. It treats the problem in the same vein as it is done for the Schrödinger operator and the related $\mathrm{KdV}(\mathrm{mKdV})$ hierarchies. The acoustic problem and the Schrödinger operator are closely connected. This connection constituted a base for the approach to the acoustic problem and the HD equation in a variety of works, see, for example, Heremah et al. [8] and Dmitrieva [5, 6]. However, as discussed below, the relation between the problems is far from being straightforward. This makes the acoustic problem interesting in its own right, and the systematic exposition of the algebraic approach to it (despite the fact that the main idea is well known and can be tracked back, for example, to Jacobi [9]) undertaken below appears to be without precedent.

The acoustic problem describes wave propagation in inhomogeneous media and, just like the Schrödinger equation, is nonintegrable for an arbitrary potential. For applications, it is important to be able to construct integrable potentials which result in solutions with given properties or asymptotic behavior. For instance, for the purposes of transmission of information, reflexionless or 
transparent potentials are of interest. These potentials are such that the problem admits solutions which asymptote to $e^{-i p x}$ as $x \rightarrow-\infty$ and $T(p) e^{i p x}$ as $x \rightarrow \infty$, with the passage coefficient $T(p) \in \mathbb{C}$ being one in absolute value. Reflexionless potentials for the acoustic problem were recently reported in a work by Novikov [13] which has drawn our attention to the problem.

The acoustic problem on the real line is described by the following ODE:

$$
\psi_{x x}=\frac{\lambda}{u^{2}(x)} \psi \equiv \lambda \epsilon(x) \psi
$$

where, say the almost everywhere positive function $\epsilon \in L_{\mathrm{loc}}^{1}(\mathbb{R})$. Here is one way how this equation arises in physics. Consider the Maxwell equations in a medium without external sources with the standard notations $(\mathbf{E}, \mathbf{H})$ for the electromagnetic field, as well as $\mathbf{D}=\epsilon \mathbf{E}$ and $\mathbf{B}=\kappa \mathbf{H}$. Suppose the medium is isotropic but inhomogeneous, that is, $\epsilon, \kappa$ are scalar quantities; suppose $\kappa \equiv 1$ and $\epsilon=\epsilon(x, y, z)$. Then

$$
[\nabla, \mathbf{B}]=\frac{1}{c} \frac{\partial \mathbf{D}}{\partial t}, \quad[\nabla, \mathbf{E}]=-\frac{1}{c} \frac{\partial \mathbf{B}}{\partial t}, \quad(\nabla, \mathbf{D})=(\nabla, \mathbf{B})=0,
$$

where $[\cdot, \cdot]$ and $(\cdot, \cdot)$ denote the vector and dot product in $\mathbb{R}^{3}$, respectively.

As usual, exclusion of the quantity $\mathbf{B}$ from (1.2) leads to an equation connecting the quantities $\mathbf{E}$ and $\mathbf{D}$ :

$$
[\nabla,[\nabla, \mathbf{E}]]=-\frac{1}{c^{2}} \frac{\partial^{2} \mathbf{D}}{\partial t^{2}}
$$

For the electric field $\mathbf{E}$, one seeks $\mathbf{E}(t ; x, y, z)=e^{i \omega t} \boldsymbol{\psi}(x, y, z)$; and taking into account the last equation of (1.2), one obtains

$$
\nabla\left(\frac{(\boldsymbol{\psi}, \nabla) \epsilon}{\epsilon}\right)+\Delta \boldsymbol{\psi}=-\frac{\omega^{2}}{c^{2}} \epsilon \boldsymbol{\psi}
$$

where $\Delta$ is a three-dimensional Laplacian.

Equation (1.1) follows if one lets $\epsilon=\epsilon(x), \boldsymbol{\psi}=(0,0, \psi(x)), \lambda=-\omega^{2} / c^{2}$, and $u^{-2}(x)=\epsilon(x)$. The dielectric permitivity $\epsilon(x)$ will be henceforth referred to as a potential.

Alternatively, one can choose $\epsilon=\epsilon(x, y)$ as well as $\boldsymbol{\psi}=(0,0, \psi(x, y))$. If this is the case, (1.4) is reduced to a linear PDE:

$$
\Delta \psi=\lambda \epsilon \psi
$$

where $\Delta$ is a two-dimensional Laplacian.

Hence, studying equations (1.1) and (1.5) is of interest for continuous media electrodynamics. A similar case can be made in acoustics, whence comes the original name of equation (1.1). Both equations describe the transmission of signals and are quite relevant for applications. 
For the Schrödinger operator, one of the most efficient ways of building potentials allowing exact solutions is the powerful and at the same time simple formal method of factorization, or the Darboux transformation, alias the dressing technique. Developing a systematic treatment in this vein for equation (1.1) appears a natural thing to do. At the outset, we present an argument illustrating the lack of one-to-one correspondence between our problem and the well-known technique for the Schrödinger equation, described, for instance, by Matveev and Salle [11], see also Shabat [14] and Svinolupov and Yamilov [15]. The basics are presented in Sections 2 and 3 of this paper. We focus primarily on various algebraic aspects of the technique, such as the related generalized Crum formulae and chains of discrete symmetries and their closing, which enable one to produce a variety of integrable potentials with desired properties.

Novikov [13] constructs a family of so-called B-potentials for the acoustic problem via a semiclassical solution ansatz. We show that these potentials naturally come up as a result of a one-step dressing on "vacuum background." B-potentials in question possess integrable point singularities, which may raise some scepticism regarding their genericity for continuous media electrodynamics. However, the dressing technique enables one to construct all sorts of potentials, including those which are neither B-potentials nor singular. We illustrate it by a single act of dressing-chain closing (in dimensions one and two) which yields regular integrable potentials (dielectric permitivity).

The acoustic problem (1.1) is also interesting from the integrable systems viewpoint. (The references quoted to this effect represent just a tiny fraction of the vast body of literature on the subject. In particular, we do not address the methods for solving the initial value problems, such as the RiemannHilbert and $\bar{\partial}$-problem techniques.) Apart from its close connection with the Schrödinger operator and the corresponding KdV (mKdV) hierarchy, investigated in detail by Dmitrieva [5, 6], it comes as a particular case of a dimensional reduction of the modified Kadomtsev-Petviashvili (mKP) equation, see Konopel'chenko and Dubrovsky [10]. It is also known to represent the Lequation of the Lax pair (or the LA pair) for the nonlinear HD PDE [5, 6, 8]. Despite apparent similarities, the algebraic relations between the equations in question are quite delicate, which often results in the impossibility of finding brute force correspondence between the solution classes of one and another. On the other hand, the dressing-chain technique appears to reveal some of the more subtle connections, see, for example Section 5 as well as the (brief) discussion around (6.7) at the end of this paper.

To this effect, we illustrate the Darboux transformation technique for ODE (1.1) by constructing an apparently new positon solution of the HD equation from a pair of its simple soliton solutions, which appears to be unaccessible within the above-mentioned techniques exploiting the connections between the HD equation and the $\mathrm{KdV}(\mathrm{mKdV})$ or $\mathrm{KP}(\mathrm{mKP})$ hierarchies. 
The above-mentioned aspect of the Darboux transformation formalism, namely, the dressing-chain closing technique, not only provides a way of finding exact solutions of nonlinear equations, but also becomes a resource for proliferation of these equations (see, e.g., Borisov and Zykov [1] or Yurov [18]), that is, building new integrable PDEs together with their LA pairs by means of the dressing-chain technique. This way of building PDE hierarchies appears to be more systematic than the technique based on the Miura transformation. As an illustration, we construct a modified Harry Dym (mHD) equation (and its LA pair) which we have not been able to find in the literature, although it has a remarkably simple form.

In Section 6 we turn to (1.5). The Moutard transformations for this equation provide a simple method for the construction of exact solutions of the Maxwell equations (1.2) with a dielectric permitivity $\epsilon=\epsilon(x, y)$, which in general has a complicated singularity structure. However, a simple periodic closing of a dressing chain, generated by the Moutard transformations, results in a regular integrable 2D dielectric permitivity.

REMARK ON EXPOSITION. In this paper, we adhere to a somewhat informal "physical" exposition style. In particular, we avoid analyzing the issue as for to which particular class one or another integrable potential or exact solution found belongs, rather emphasizing on the consequences of the purely algebraic or discrete symmetry structure of the method. For a state-of-the-art rigorous mathematical exposition of the Darboux transformation technique for general Sturm-Liouville operators, see, for example, Gesztesy and Teschl [7]. The discrete symmetry is essentially based on a single formula (2.9); simple as it may be, it has remarkable consequences.

2. Discrete symmetries of the one-dimensional acoustic problem. The Darboux transformation, namely, the so-called single commutation technique, was set forth in Darboux [4] and considerably developed by Crum [3]. It is based on an observation which makes use of the existence of specific discrete isospectral symmetries of the equation underlying the Sturm-Liouville operator and is standard in the theory of integrable PDEs, see, for instance, $[11,14]$ and the references therein.

Dealing with (1.1), one starts out with a well-known nonlinear substitution owing to which the solutions of (1.1) are usually obtained in the parametric form $[5,6]$. Let

$$
u(x)=v_{y}(y), \quad x=v(y) .
$$

This reduces equation (1.1) to

$$
\psi_{y y}=U \psi_{y}+\lambda \psi
$$


with $U=v_{y y} / v_{y}$. The above quantity $U(y)$ will be referred to as a potential as well as the dielectric permitivity function $\epsilon(x)=1 / u^{2}(x)$ mentioned earlier.

To this effect, we would like to emphasize the following two important issues.

(1) In spite of the fact that (2.2) is easily reducible to the Shrödinger operator, from the point of view of finding integrable potentials, this connection is not trivial. We address it in more detail.

A formal substitution

$$
\psi \rightarrow \sqrt{v_{y}} \psi
$$

transforms (2.2) into the stationary Schrödinger equation

$$
\psi_{y y}=(\lambda+V(y)) \psi
$$

where the potential $V(y)$ is related to the potential $U(y)$ of the acoustic problem (2.2) via

$$
V=\frac{U^{2}-2 U_{y}}{4}
$$

Linearizing (2.5) with a substitution $U=-2 p_{y} / p$, one sees that $p(y)$ in turn satisfies (2.4) with $\lambda=0$.

The Darboux transformation for (2.4) is well known, and, at the first sight, it may appear that developing an independent technique for the acoustic problem (2.2) is superfluous, as one can generate integrable potentials $V$ for the Schrödinger operator. However, integration of the Ricatti equation (2.5) presents a problem of its own, and the following argument shows that there is no one-to-one correspondence between problems (2.2) and (2.4), as far as the potentials are concerned.

Indeed, let $U(y)$ be a specific potential for the acoustic problem (2.2) not depending on any free parameters. From (2.5), one can (uniquely) get the Shrödinger potential $V(y)$ and further substitute it into (2.4). In order to reconstruct the initial potential $U(y)$, (2.4) should be solved with $\lambda=0$. Let the solution be $p=p\left(y, C_{1}, C_{2}\right)$ depending on a pair of constants $C_{1}, C_{2}$. One of these constants, say $C_{1}$, plays the normalizing role corresponding to scaling $p \rightarrow C_{1} p$ and can be omitted. However, the restored potential $U=U\left(y, C_{2}\right)$ will inevitably depend not only on $y$, but on the free parameter $C_{2}$ as well. Hence, a single potential in the Shrödinger operator generates the whole family of potentials for the acoustic problem, and in order to single out a specific potential for the latter, one would have to subsequently develop some selection mechanism by studying the sequence of maps $U(y) \rightarrow V(y) \rightarrow U\left(y, C_{2}\right) \rightarrow U(y)$. It is not clear how to do it; on the other hand, such a necessity gets bypassed if one develops the Darboux transform formalism directly apropos of the operator (2.2) without using (2.4). The simple procedure is exposed below in the form 
suitable for exposing the main results of this paper, which are various new families of integrable potentials.

(2) On the other hand, (2.2) arises as a result of dimensional reduction of the L-equation for the $2+1 \mathrm{mKP}$ equation, that is, by substituting $\psi(z, y) \rightarrow$ $e^{-(\lambda / \sigma) z} \psi(y)$ into

$$
\sigma \psi_{z}+\psi_{y y}+V \psi_{y}=0
$$

The mKP equation was studied in depth by Konopel'chenko and Dubrovsky [10] as an initial-value problem within the nonlocal Riemann-Hilbert and $\bar{\partial}$ dressing techniques and the Miura transformation between the equations mKP and KP. A variety of integrable potentials, including the transparent ones, was constructed in [10]. However, these potentials do not appear amenable to the dimensional reduction in question, namely, the present work gives a considerably more general way to integrate the acoustic problem and HD equation (in the same fashion the solutions of the mKP equation obtained in [10] do not generate the solutions for the HD equation in a tangible way). On the other hand, the results of this paper can certainly be interpreted as plane solutions of (2.6).

Following Shabat [14], we seek elementary discrete symmetries of (2.2) effecting the change

$$
\psi \longrightarrow \psi^{(1)}=f \psi_{y}+g \psi
$$

for some $\lambda$-independent functions $f$ and $g$ of $y$.

One easily verifies that there are three formal distinct discrete symmetries of the type (2.7) for (2.2). They are

$$
\begin{gathered}
\psi \longrightarrow \psi^{(1)}=\frac{\psi}{v}, \quad v \longrightarrow v^{(1)}=\frac{1}{v}, \\
\psi \longrightarrow \psi^{(1)}=\frac{\psi_{y}}{v_{y}}, \quad v \longrightarrow v^{(1)}=\int \frac{d y}{v_{y}}, \\
\psi \longrightarrow \psi^{(1)}=\frac{\psi_{1} \psi_{y}}{\psi_{1, y}}-\psi, \\
v_{y} \longrightarrow v_{y}^{(1)}=v_{y}\left(\frac{\psi_{1}}{\psi_{1, y}}\right)^{2}, \\
U \longrightarrow U^{(1)}=U+2 D \ln \frac{\psi_{1}}{\psi_{1, y}} .
\end{gathered}
$$

In the latter equation, $\psi_{1}=\psi_{1}\left(y, \lambda_{1}\right)$ is a particular solution of (2.2) with the spectral parameter value $\lambda_{1}$, further referred to as a prop solution $D=\partial_{y}$ and $\psi_{1, y}=D \psi_{1}$. We assume, following the above remark on exposition, that all the necessary positivity or monotonicity properties of the quantities $u, v, \psi_{1}$, and so forth are satisfied in order that the final expressions not be ill defined, as it is the case in the series of ensuing examples. 
The former two symmetries (2.8) define the new quantity $U^{(1)}=v_{y y}^{(1)} / v_{y}^{(1)}$ in a way independent of any solution $\psi(y, \lambda)$ of (2.2). These symmetries arise as a particular case of (2.7) as the result of gauging corresponding to the choice of $f$ or $g$ alternatively zero. These symmetries have a trivial kernel in the solution space of (2.2). According to the terminology of [14], we call the symmetries (2.8) T-symmetries, sometimes referred to as Schlesinger transforms. (In the context of soliton solutions, the T-symmetries play the part of explicitly invertible Bäcklund transforms, see, e.g., [15].) On the other hand, the transformation (2.9) alias the Darboux transformation, which [14] calls an S-symmetry, does have a nontrivial kernel on the solution space of (2.2) (one can let $\psi=\psi_{1}$ in the first equation of (2.9) and get zero). This property thereof is essential.

We use the common term dressing for the application of transformation (2.9) to a triple $(\psi, v, U)$, with the resulting triple $\left(\psi^{(1)}, v^{(1)}, U^{(1)}\right)$ being referred to as the dressed one. Despite a nearly trivial countenance, the Darboux transform (2.9) in principle enables one to engineer potentials with arbitrary discrete spectra ad hoc. Indeed, suppose it is possible to solve (2.2) formally (namely, obtaining among others some "nonphysical" solutions unbounded as $|y| \rightarrow \infty)$ for some potential $U$ and all $\lambda \in \mathbb{R}$. Suppose $\psi_{1}\left(y, \lambda_{1}\right)$ is such a solution. We denote its linearly independent counterpart as $\hat{\psi}_{1}\left(y, \lambda_{1}\right)$, that is,

$$
\hat{\psi}_{1}=\psi_{1} \int d y \frac{v_{y}}{\psi_{1}^{2}} .
$$

Dressing $\hat{\psi}_{1}$ according to (2.9), we find

$$
\hat{\psi}_{1}^{(1)}=\frac{v_{y}}{\psi_{1, y}} .
$$

Therefore, if one comes up with a nonphysical prop solution $\psi_{1}$ by requiring that its derivative $\psi_{1, y}$ be strictly positive and rapidly growing as $|y| \rightarrow \infty$, then, in the spectrum of the dressed potential $U^{(1)}$, there will appear a level $\lambda_{1}$ not present in the original spectrum for $U$. Since the principle for the choice of the value of $\lambda_{1}$ is such that this value is not to be present in the physical spectrum for $U$, repeating the dressing procedure $n$ times will result in a potential $U^{(n)}$ possessing $n$ new prechosen levels $\lambda_{j}, j=1, \ldots, n$. See (3.6), (3.7), and (3.8) in the sequel.

Conversely, the function $\hat{\psi}_{1}^{(1)}$ generates an inverse transformation (undressing) to (2.9). Thus, one can as well remove some prechosen levels from the spectrum of a potential.

3. Crum formulae and dressing chains for the classical acoustic spectral problem. Below we present the formulae describing an $n$-step dressing procedure for any $n \in \mathbb{N}$, whose analogues are known for the Schrödinger equation as the Crum formulae [3]. We derive the target formulae (3.6), (3.7), and (3.8) for (2.2) following the procedure exposed in [17]. 
A single act of dressing (2.9) can be iterated $n$ times to yield a triple $\left(\psi^{(n)}\right.$, $\left.v^{(n)}, U^{(n)}\right)$. One starts out by dressing a triple $(\psi, v, U) \equiv\left(\psi^{(0)}, v^{(0)}, U^{(0)}\right)$ corresponding to a spectral parameter $\lambda$ with a prop function $\psi_{1} \equiv \psi_{1}^{(0)}$, which is a formal solution of (2.2) with a spectral parameter $\lambda_{1}$ and a potential $U^{(0)}$. The resulting solution $\psi^{(1)}$ solves (2.2) with the dressed potential $U^{(1)}$ (and the same spectral parameter $\lambda$ ). On the $j$ th step, $j=1, \ldots, n$, one uses some prop solution $\psi_{j}^{(j-1)}$ which solves (2.2) with a predressed potential $U^{(j-1)}$ and a spectral parameter value $\lambda_{j}$ to produce the $j$ times dressed solution $\psi^{(j)}$ and the potential $U^{(j)}$ (as well as the function $v^{(j)}$ with $U^{(j)}=v_{y y}^{(j)} / v_{y}^{(j)}$ ). Note that the spectral parameter $\lambda$ in the dressed equations for $\psi^{(j)}$ is the same for all $j=1, \ldots, n$.

It is easy to see that the $n$ times dressed solution $\psi^{(n)}$ will have the form

$$
\psi^{(n)}=\sum_{j=1}^{n} a_{j} D^{j} \psi+(-1)^{n} \psi,
$$

with the functions coefficients $a_{j}$ to be found, which of course will depend on the choice of the prop solutions $\psi_{j}^{(j-1)}$. It follows from (2.9) that

$$
U^{(n)}=U+2 D \ln a_{n},
$$

for

$$
\begin{aligned}
& \psi^{(n)}=\prod_{j=1}^{n} \frac{\psi_{j}^{(j-1)}}{D \psi_{j}^{(j-1)}} D^{n} \psi^{(0)}+\cdots+(-1)^{n} \psi^{(0)}, \\
& U^{(n)}=U^{(0)}+2 D \ln \prod_{j=1}^{n} \frac{\psi_{j}^{(j-1)}}{D \psi_{j}^{(j-1)}},
\end{aligned}
$$

where the ellipses in the first formula stand for the terms containing the derivatives of $\psi^{(0)}$ of orders from 1 through $n-1$.

So far, the choice of the prop solutions $\psi_{i}^{(i-1)}$ has been quite arbitrary. But suppose now that the original equation (2.2) possesses $n$ distinct formal solutions $\psi_{j}$ corresponding to spectral parameter values $\lambda_{j}, j=1, \ldots, n$. Let $\psi_{j} \equiv \psi_{j}^{(0)}$ and consider the following dressing procedure (which will be further used for the dressing chain construction):

$$
\begin{array}{ccccccc}
\psi^{(0)} & \boldsymbol{\psi}_{1}^{(\mathbf{0})} & \psi_{2}^{(0)} & \cdots & \psi_{n-1}^{(0)} & \psi_{n}^{(0)} & U^{(0)} \\
\psi^{(1)} & 0 & \boldsymbol{\psi}_{2}^{(\mathbf{1})} & \cdots & \psi_{n-1}^{(1)} & \psi_{n}^{(1)} & U^{(1)} \\
\vdots & & & & & & \vdots \\
\psi^{(n-1)} & 0 & 0 & \cdots & 0 & \boldsymbol{\psi}_{\mathbf{n}}^{(\mathbf{n}-\mathbf{1})} & U^{(n-1)} \\
\psi^{(n)} & 0 & 0 & \cdots & 0 & 0 & U^{(n)} .
\end{array}
$$


Namely, for $j=1, \ldots, n$ on the above diagram (3.4), every new line $j+1$ is obtained by dressing the functions from the preceding line $j$ by (2.9) with a prop solution $\boldsymbol{\psi}_{\mathbf{j}}^{(\mathbf{j}-\mathbf{1})}$ marked in bold.

Zeroes, proliferating as one moves down the diagram, stem from the nontrivial kernel property of the S-symmetry, and it is this property that now enables one to find the unknown functions $a_{j}$. Indeed, substitution of any $\psi_{j}=\psi_{j}^{(0)}$ for $\psi$ in the right-hand side of (3.1) will yield zero. Hence, the coefficients $a_{j}$ satisfy a system of $n$ independent linear algebraic equations, namely,

$$
\sum_{k=1}^{n} a_{k} D^{k} \psi_{j}+(-1)^{n} \psi_{j}=0, \quad j=1, \ldots, n .
$$

Solving it by the Kramer rule and substituting the result into (3.1) and (3.2), we end up having

$$
U^{(n)}=U+2 D \ln \frac{\tilde{\Delta}_{n}}{\Delta_{n}}
$$

that is,

$$
v_{y}^{(n)}=v_{y}\left(\frac{\widetilde{\Delta}_{n}}{\Delta_{n}}\right)^{2}, \quad \psi^{(n)}=\frac{\widehat{\Delta}_{n+1}}{\Delta_{n}},
$$

where $\Delta_{n}$ and $\widetilde{\Delta}_{n}$ are determinants of square $n \times n$ matrices, whereas $\widehat{\Delta}_{n+1}$ of an $n+1 \times n+1$ matrix as follows:

$$
\begin{aligned}
\Delta_{n} & =\left|\begin{array}{ccc}
D \psi_{1} & \cdots & D^{n} \psi_{1} \\
\vdots & & \vdots \\
D \psi_{n} & \cdots & D^{n} \psi_{n}
\end{array}\right|, \\
\tilde{\Delta}_{n} & =\left|\begin{array}{ccc}
\psi_{1} & \cdots & D^{n-1} \psi_{1} \\
\vdots & & \vdots \\
\psi_{n} & \cdots & D^{n-1} \psi_{n}
\end{array}\right|, \\
\widehat{\Delta}_{n+1} & =\left|\begin{array}{ccc}
\psi & \cdots & D^{n} \psi \\
\psi_{1} & \cdots & D^{n} \psi_{1} \\
\vdots & & \vdots \\
\psi_{n} & \cdots & D^{n} \psi_{n}
\end{array}\right| .
\end{aligned}
$$

Note that the linearity of (2.2) makes the choice of the sign before $\psi^{(n)}$ irrelevant. The obtained formulae (3.6), (3.7), and (3.8) make it possible to find rich families of exact solutions of (1.1).

EXAMPLE 3.1 (reflexionless potentials). The easiest case is dressing from the birthday suit, or on the vacuum background, assuming $U=0$ (hence, $x=$ $c_{1} y+c_{2}, u(x)=c_{1}$, where $c_{1}$ and $c_{2}$ are arbitrary constants). Such a natural 
rigging yields gratis all the B-potentials reported by Novikov [13]. Moreover, the formulae for their computation derived therein turn out to be particular cases of (3.6), (3.7), and (3.8) with merely $v_{y}=1$. For instance, $\psi_{1}=\sinh \xi_{1}$ and $\psi_{2}=\cosh \xi_{2}$ (here $n=2, y_{j}$ are constants, $\xi_{i}=k_{j}\left(y-y_{j}\right)$, and $j=1,2$ ) yield a reflexionless B-potential with a power $2 / 3$ singularity: $\epsilon^{(2)}=1 /\left[u^{(2)}\right]^{2}$, where

$$
u^{(2)}=\left(\frac{k_{2} \sinh \xi_{1} \sinh \xi_{2}-k_{1} \cosh \xi_{1} \cosh \xi_{2}}{k_{1} \sinh \xi_{1} \sinh \xi_{2}-k_{2} \cosh \xi_{1} \cosh \xi_{2}}\right)^{2} .
$$

The same $\psi_{1}$ and $\psi_{2}=\sinh \xi_{2}$ yield another potential with a power $4 / 5$ singularity:

$$
u^{(2)}=\left(\frac{k_{2} \sinh \xi_{1} \cosh \xi_{2}-k_{1} \sinh \xi_{2} \cosh \xi_{1}}{k_{1} \sinh \xi_{1} \cosh \xi_{2}-k_{2} \sinh \xi_{2} \cosh \xi_{1}}\right)^{2} .
$$

(Note that the expressions for $u^{(2)}$ are parametric. In order to interpret the formulae correctly, the reader is referred back to (1.1), (2.1), and (2.2). The orders of the singularities pertain to the potential $\epsilon(x)$, which is a zero of the function $u(x)$ and a singularity of the potential $U(y)=v_{y y} / v_{y}$, where the function $v(y)$ solves the equation $u[v(y)]=v_{y}(y)$.) By construction, these potentials have only two levels $\lambda_{1,2}$.

In addition, all the regular reflexionless potentials can be also built by formulae (3.6), (3.7), and (3.8) once again by dressing $U=0$. Indeed, the passage coefficient for a regular $n$-level reflexionless potential can be expressed by a well-known formula

$$
T_{n}(p)=\prod_{j=1}^{n} \frac{k_{j}-i p}{k_{j}+i p}
$$

with $\lambda_{j}=k_{j}^{2}$. As has been pointed out earlier, the levels $\lambda_{j}$ can be successively removed from the spectrum by means of the inverse of the Darboux transformation (still having the form (2.9)), each application of which will kill a term in the product. Successively applying this procedure $n$ times for the passage coefficients, we have

$$
T_{n}(p) \longrightarrow T_{n-1}(p) \longrightarrow \cdots \rightarrow T_{0}(p)=1
$$

This proves our assertion because the case $R(p)=0$ and $T(p)=1$ for the reflection and the passage coefficient for all $p$ is feasible with $U=0$ only. It is worth reiterating the point that the above argument owes itself to the fact that the S-symmetry possesses a nontrivial kernel in the space of solutions of (2.2).

Matveev and Salle [11] find super-reflexionless potentials for the KdV equation, alias positons. In the same vein, one can operate on (1.1) and (2.2). In order 
to do so, one should use formulae (3.6), (3.7), and (3.8) with $n=2$ choosing the prop solutions $\psi_{1,2}$, respectively, as $\psi_{1}\left(y, \lambda_{1}\right)$ and $\psi_{1}\left(y, \lambda_{1}+\delta\right)$, and then letting $\delta \rightarrow 0$. If $U=0$ and $\psi_{1}$ generates a single soliton potential, then (3.6) and (3.7) define a single positon potential. Section 4 describing the positon solutions of the HD equation contains the aforementioned computation.

In addition to B-potentials, various other interesting ones can be produced. For instance, one can construct soluble potentials with a finite equidistant spectrum. (The same statement applies to the (stationary) Schrödinger equation.)

One can also investigate potentials which change in a specific simple way under the Darboux transform, for example, such that $U \rightarrow U+$ const or $U \rightarrow$ const $U$. For the Schrödinger equation, the former transformation is shape invariant and for $n=1$, it results in the harmonic oscillator potential. We develop an analogue for the model (1.1) and (2.2) under investigation.

EXAMPLE 3.2 (shape-invariant potential). Let

$$
U^{(1)}=U+\frac{2}{\omega^{2}},
$$

for a constant $\omega$. Then we can parametrically obtain the function $u(x)$ from (1.1) as follows:

$$
u(x)=\alpha z \exp \left(-\omega^{2} z-\frac{\kappa^{2}}{z}\right), \quad x=x_{0}-\alpha \omega^{2} \int d z \exp \left(-\omega^{2} z-\frac{\kappa^{2}}{z}\right),
$$

where, $\kappa, x_{0}$, and $\alpha$ are real constants and $z=\exp \left(-y / \omega^{2}\right)$. The prop function $\psi_{1}$ rendering the potential $U^{(1)}$ from $U$ has the countenance $\psi_{1}=\exp \left(-\omega^{2} z\right)$ and solves (2.2) with an eigenvalue $\lambda_{1}=b^{2} / \omega^{2}$. It is easy to verify that the dielectric permitivity $\epsilon(x)=1 / u^{2}(x)$ has a second-order pole at $x=x_{0}$.

The theory of the Darboux transformation for the Schrödinger equation utilizes the concept of dressing chains of discrete symmetries and their closing. The work of Veselov and Shabat [16] elucidates how the dressing-chain closing method can be used in order to obtain various potentials with meaningful mathematical physics. Namely, a simple closing procedure leads one to the harmonic oscillator potential (resulting also in a shape-invariant change of potential). A more complicated closing scheme results in finite-gap potentials as well as the fourth and the fifth Peinleve equations, see [16].

Dressing chains can be written out for (2.2) as well. We introduce a sequence $\left\{f_{n}\right\}_{n \geq 1}$ of functions as follows:

$$
f_{n}=D \ln \psi_{n}^{(n-1)},
$$

with the quantity $\psi_{n}^{(n-1)}$ as it has been introduced in diagram (3.4) (where it appeared in bold). In particular, it corresponds to the pre-chosen value $\lambda_{n}$ of the spectral parameter. 
One can verify by hand, starting from $n=1$, that

$$
U^{(n)}=U-2 D \ln \prod_{j=1}^{n} f_{j} .
$$

Besides, direct substitution shows that $f_{n}$ satisfies the equation

$$
f_{n}^{\prime}+f_{n}^{2}-U^{(n)} f_{n}=\lambda_{n},
$$

where $f^{\prime}=D f$. The two latter relations imply the recursion connecting $f_{n}$ and $f_{n+1}$ as follows:

$$
\left(f_{n} f_{n+1}\right)^{\prime}=f_{n} f_{n+1}\left(f_{n}-f_{n+1}\right)+\lambda_{n+1} f_{n}-\lambda_{n} f_{n+1} .
$$

Equation (3.18) represents a dressing chain for the acoustic problem.

In a way analogous to the theory of dressing chains for the Schrödinger equation [16], we are interested in T-periodic chain closing, namely, imposing the condition $f_{n+T}=f_{n}$ for an integer $T \geq 1$. We will consider here the easiest case $T=1$.

EXAMPLE 3.3 (regular potential). Given the spectral parameter values $\lambda_{1,2}$, one obtains a one-parameter family of potentials indexed by a constant $c$ :

$$
U=\frac{-\left(\lambda_{1}-\lambda_{2}\right)^{2} y^{2}+2 c\left(\lambda_{2}-\lambda_{1}\right) y+6 \lambda_{1}-2 \lambda_{2}-c^{2}}{2\left[\left(\lambda_{1}-\lambda_{2}\right) y+c\right]} .
$$

If $\lambda_{2}=3 \lambda_{1}>0$ and $c=0$, we can express the function $u(x)$ parametrically:

$$
x(y)=\frac{\sqrt{\pi}}{2 \alpha} \operatorname{Erf}(\alpha y), \quad u(y)=\exp \left(-\alpha^{2} y\right)
$$

where $\alpha^{2}=-\lambda / 2>0$.

It is known that for the Schrödinger equation, a nontrivial chain closing operation with $T>1$ results in finite gap potentials [16]. Such potentials for the HD equation are due to Dmitrieva [5]. A close analogy between the Schrödinger equation and the acoustic problem (see $[2,6,8]$ ) suggests that one can expect results similar to those of [16] apropos of the analysis of higher-order chain closing for $T>1$. We expect that potentials built in such a way can have interesting physical applications, such as a model of wave propagation in media whose dielectric permitivity is a periodic function of a single spatial variable.

4. HD equation. The $1+1 \mathrm{HD}$ equation

$$
u_{t}=u^{3} u_{x x x}+\beta u_{x}
$$

with some real constant $\beta$, has been studied quite extensively since the late seventies, see, for example, [5, 6, 8] and the references therein. It arises in the study 
of evolution equations solvable via the spectral transforms method based on the string rather than the Schrödinger equation. The principal approach to it has been based on its relation to the $\mathrm{KdV}, \mathrm{mKdV}$, and other more classical hierarchies of integrable PDEs [5, 6]. However, as was mentioned above, this relation is not straightforward, and the direct approach developed herein in principle enables one to produce a wider range of solutions of the HD equation. As an example, we construct a simple positon solution below.

The acoustic problem (1.1) is the first equation in the LA pair for the HD equation (4.1), with the full pair being

$$
\psi_{x x}=\frac{\lambda}{u^{2}} \psi, \quad \psi_{t}=(4 \lambda u+\beta) \psi_{x}-2 \lambda u_{x} \psi
$$

The coordinate change (2.1) in the presence of time dependence becomes

$$
t \rightarrow t, \quad x \longrightarrow v(y, t)
$$

thus

$$
\partial_{x} \rightarrow \frac{1}{v_{y}} \partial_{y}, \quad \partial_{t} \rightarrow \partial_{t}-\frac{v_{t}}{v_{y}} \partial_{y}
$$

After this change, (4.2) becomes

$$
\psi_{y y}=\frac{v_{y y}}{v_{y}} \psi_{y}+\lambda \psi, \quad \psi_{t}=\left(\frac{v_{t}+\beta}{v_{y}}+4 \lambda\right) \psi_{y}-\frac{2 \lambda v_{y y}}{v_{y}} \psi
$$

and the HD equation (4.1) transforms to

$$
v_{y}\left(v_{t} v_{y y}-v_{y t} v_{y}\right)+3 v_{y y}^{2}+v_{y}\left(v_{4 y} v_{y}-4 v_{3 y} v_{y y}+\beta v_{y y}\right)=0
$$

with the notations $v_{3 y}$ and $v_{4 y}$ for the partial derivatives in $y$ of order 3 and 4 , respectively.

The goal now is to extend the Darboux transformation (2.9) for (2.2) alias the first equation in (4.5) so that it agrees with the second equation in the LA pair. One just includes the $t$-dependencies in (2.9). At this point, it only provides the value of the partial derivative

$$
v_{y}^{(1)}=\left(\frac{\psi_{1}}{\psi_{1, y}}\right)^{2} \equiv A(y, t)
$$

rather than the dressed quantity $v^{(1)}(y, t)$ of interest. 
LEMmA 4.1. The Darboux transformation (2.9), (4.9) is an S-symmetry for the LA-pair (4.5), and therefore for the HD equation (4.6).

Proof. Let $v_{t}^{(1)}=B(y, t)$ be unknown and we assume that $v^{(1)}$ satisfies the second equation of the pair (4.5) with the function $\psi^{(1)}$ dressed according to (2.9), where the quantities $(\lambda, \beta)$ remain the same. One can express the unknown quantity $B$ as follows:

$$
B=\left(\frac{\psi_{1}}{\psi_{1, y}}\right)^{2}\left(\beta+4 \mu v_{y}+v_{t}-2 v_{y} U_{y}\right)+\frac{4 \psi_{1} v_{y y}}{\psi_{1, y}}-4 v_{y}-\beta,
$$

and verify that $B_{y}=A_{t}$. It follows that

$$
v^{(1)}(y, t)=\int A d y+B d t
$$

with a closed one-form under the integral.

This enables one to construct exact solutions for this equation.

EXAMPLE 4.2 (single soliton solution and positon solution). Let $v=y, \lambda_{1}=$ $k^{2}$, and $\psi_{1}(y, t, k)=\sinh [\phi(y, t, k)]$ with $\phi=k\left(y+\left(4 k^{2}+\beta\right) t\right)$. Then, by (4.9), one has

$$
v^{(1)}=\frac{1}{k^{3}}(\phi-\tanh \phi)-(4+\beta) t
$$

The function $v^{(1)}(y, t, k)$ determines a single soliton B-potential

$$
U^{(1)}=\frac{v_{y y}^{(1)}}{v_{y}^{(1)}}
$$

mentioned in Section 3.

The single positon potential is obtained from two distinct soliton solutions $\psi_{1}(y, t, k)$ and $\psi_{1}(y, t, k+\delta)$, used as the prop functions $\psi_{1,2}$ in formulae (3.6), (3.7), and (3.8) with $n=2$, taking the limit as $\delta \rightarrow 0$; namely,

$$
v_{y}^{(2)}=v_{y}\left(\frac{\psi_{1, y y k} \psi_{1, y}-\psi_{1, y y} \psi_{1, y k}}{\psi_{1, y k} \psi_{1}-\psi_{1, y} \psi_{1, k}}\right)^{2}
$$

where the subscript $k$ means differentiation by $k$. Taking $\psi_{1}$ explicitly as the hyperbolic sine in the previous example results in

$$
v_{y}^{(2)}=k^{4}\left(\frac{\sinh (2 \phi)+2 \tilde{\phi}}{\sinh (2 \phi)-2 \tilde{\phi}}\right)^{2}
$$

with $\tilde{\phi}=k\left(y+\left(12 k^{2}+\beta\right) t\right)$. 
5. Modified HD equation. It is well known that the dressing formalism enables one to produce hierarchies of integrable PDEs. Borisov and Zykov [1] proposed a technique for proliferation of integrable equations which they applied to the KdV and the Sine-Gordon (SG) equations. The technique is based on the discrete symmetries dressing-chain closing. The main idea of the approach is as follows. The equation (for illustration purposes we take the KdV equation) is written as a compatibility condition of a pair of equations, further denoted as $L_{1}$ and $A_{1}$. Each of these equations is quadratic in the auxiliary field. Using invariance of the pair with respect to the Darboux transformation (which is viewed as a discrete symmetry), a second pair $L_{2}$ and $A_{2}$ of equations is built. Excluding the potentials from $L_{1}$ and $L_{2}$, and $A_{1}$ and $A_{2}$, it is possible to obtain two equations, which Borisov and Zykov [1] call an $x$ and a $t$ chain, respectively. (We further use the notations $C_{x}$ and $C_{t}$ instead.) If a potential is excluded from $L_{1}$ and $A_{1}$, one ends up with an mKdV equation. The equations $C_{x}$ and $C_{t}$ can be converted into the LA pair for the $\mathrm{mKdV}$ equation in two ways, with the Darboux transformation being already known.

This procedure can be repeated, producing new equations with their LA pairs. In this vein, the $\mathrm{m}^{2} \mathrm{KdV}$ and $\mathrm{m}^{3} \mathrm{KdV}$ equations were obtained. The former becomes the exponential Calogero-Degasperis equation [2] after an exponential change, the latter contains an elliptic equation of the same authors.

In spite of its simplicity, the technique described is very powerful. This can be illustrated by the following examples. First it was claimed in Mikhailov et al. [12] that the $\mathrm{m}^{N} \mathrm{KdV}$ equations with $N=0, \ldots, 3$, together with the KricheverNovikov equation, exhaust (modulo a contact transformation) all the integrable equations of the form $u_{t}+u_{x x x}+f\left(u_{x x}, u_{x}, u\right)=0$. Second, applying their approach to the SG equation, Borisov and Zykov [1] have succeeded in coming up with a new nonlinear equation already on the second step. This equation has a nontrivial Bäcklund transform, admitting an interesting $2 \pi$-kink-shelf solution.

The same technique was shown to be applicable to the study of considerably more difficult $(1+2)$-dimensional nonlinear PDEs. For instance, in [18], the proliferation procedure was successfully adapted to the Kadomtsev-Petviashvili and Boiti-Leon-Pempinelli equations.

We apply this formalism to the HD equation. First note that the LA pair for (4.6) can be written as a system of two Ricatti equations:

$$
\begin{gathered}
g_{y}=-\lambda g^{2}-U g+1 \\
g_{t}=\lambda\left(2 U_{y}-\frac{v_{t}+\beta}{v_{y}}-4 \lambda\right) g^{2}-\left(\frac{v_{t y}}{v_{y}}+4 \lambda U\right) g+\frac{v_{t}+\beta}{v_{y}}+4 \lambda .
\end{gathered}
$$

The second summand in the right-hand side of the second equation has a term, denoted as $v_{t y}$, representing a fairly long expression which can be derived from (4.6). The function $g=g(y, t)$ is connected with the solution $\psi$ of (4.5) as $g=\psi_{y} / \psi$. Excluding the function $v$ from (5.1) and returning to the old 
variables via $x=g$ and $u=g_{y}$, we obtain an mHD equation:

$$
\begin{aligned}
u_{t}= & u^{3} u_{3 x}+3 u^{2} u_{x} u_{x x}-3 \lambda^{2} x u^{2} \\
& -\frac{3 u^{2}\left(u u_{x x}+u_{x}^{2}\right)}{x}+\frac{6 u^{3} u_{x}}{x^{2}}+\frac{3 u^{2}\left(1-u^{2}\right)}{x^{3}} .
\end{aligned}
$$

(By analogy with the mKdV equation in [1], we call (5.2) the mHD equation.) As one can see, this equation has a different countenance than the HD equation. However, omission of all the summands but the first one in the right-hand side of (5.2) yields the HD equation (4.1) with $\beta=0$. Note that (5.2) can be rewritten quite nicely in new variables $x=1 / z, u(x, t)=\sqrt{\theta(z, t)}$ :

$$
\left(\theta^{-1 / 2}\right)_{t}=\frac{3 \lambda^{2}}{z}+z^{3}\left(\frac{1}{2} z^{3} \theta_{3 z}+\frac{9}{2} z^{2} \theta_{z z}+9 z \theta_{z}+3 \theta-3\right) .
$$

The formula (5.3) can be simplified even further by changing

$$
\theta=e^{-2 \xi} \eta(\xi, t)+1, \quad y=\log z .
$$

As the result, it becomes

$$
\left[\left(e^{-2 \xi} \eta+1\right)^{-1 / 2}\right]_{t}=3 \lambda^{2} e^{-\xi}+\frac{1}{2} e^{\xi}\left(\eta_{3 \xi}-\eta_{\xi}\right) .
$$

However, we will be considering the mHD equation in the form (5.3). Note that in the stationary $\theta_{t}=0$ case, it reduces to a linear ODE!

The dressing chain method produces not only (5.3), but also its LA pair. It is constructed as follows. Return to the chain (3.18) and let $f_{n}=1 / \mathcal{g}, f_{n+1}=\Psi$, $\lambda_{n}=\lambda$, and $\lambda_{n+1}=\mu$. Considering $\mu$ as a spectral parameter, one can see that (3.18) can be viewed as an L-equation of the LA pair for (5.3). One should also define the second, nonstationary chain $C_{t}$ for the functions $g_{n}$ (in terms of the dynamical equation in (4.5)) and build the A-equation. Omitting the lengthy but straightforward computation, we present the LA pair for (5.3), written in the variables $t$ and $z$ :

$$
\begin{gathered}
\Psi_{z}=\frac{\mu}{z^{2} \sqrt{\theta}} \Psi^{2}+\left(\frac{1}{z}+\frac{1}{z \sqrt{\theta}}-\frac{\mu}{z^{3} \sqrt{\theta}}\right) \Psi-\frac{1}{z^{2} \sqrt{\theta}}, \\
\Psi_{t}=\mu a \Psi^{2}+b \Psi+c,
\end{gathered}
$$

where

$$
\begin{aligned}
a= & -4 \mu+2 \lambda-\frac{\lambda^{2}}{z^{2}}-2 \lambda \sqrt{\theta}+(\theta-1) z^{2}+2 z^{3} \theta_{z}+\frac{1}{2} z^{4} \theta_{z z}, \\
b= & 4\left(\frac{\lambda}{z}-z-z \sqrt{\theta}\right) \mu+\frac{1}{2} z^{5} \theta_{z z}+2 z^{4} \theta_{z}+\left(\theta-\frac{\lambda}{2} \theta_{z z}-1\right) z^{3} \\
& -3 \lambda z^{2} \theta_{z}+3 \lambda(1-\theta) z-\frac{3 \lambda^{2}}{z}+\left(\frac{\lambda}{z}\right)^{3}, \\
c= & 4 \mu-\frac{1}{2} z^{4} \theta_{z z}-3 z^{3} \theta_{z}+(1-3 \theta-2 \sqrt{\theta}) z^{2}-2 \lambda+\left(\frac{\lambda}{z}\right)^{2} .
\end{aligned}
$$


Note that the spectral parameter in (5.6) is $\mu$, whereas $\lambda$ enters the nonlinear equation (5.3). As one can see, the LA pair for (5.3) also has the form of a pair of Ricatti equations. These equations can be simultaneously linearized in order to represent (5.3) as a compatibility condition for two linear equations, as it is done in the theory of solitons.

We will not proceed further with the mHD equation. To conclude this section, we would like to repeat the statement that the exact solutions of (5.3) can easily be found via the dressing technique, and this procedure can be extended in order to produce the mHD equation and its LA pair. It is worth emphasizing that equations HD and (5.3) are members of different hierarchies. Discrete symmetries enable one to establish connections between different integrable equation hierarchies in a way apparently more systematic than trying to guess the Miura transformation.

6. Moutard transformations. We devote this last section to (1.5) which has been obtained from the Maxwell equations (1.2) in the case of an isotropic but inhomogeneous in two directions $(x, y)$ medium.

Clearly, a PDE (1.5) is harder to investigate than an ODE (1.1). Nevertheless, its analysis in terms of the Darboux transform (2.9), known also as the Moutard transformation [11], is quite similar to its ODE cousin. Below, we present the relevant formulae without the derivation details.

LEMMA 6.1. Let $\psi=\psi(x, y)$ and $\phi=\phi(x, y)$ be two particular solutions of (1.5), that is,

$$
\Delta \psi-\lambda \epsilon \psi=\Delta \phi-\lambda \epsilon \phi=0
$$

The following transformations represent an analogue of (2.9):

$$
\psi \longrightarrow \psi^{(1)}=\frac{\theta[\psi, \phi]}{\phi}, \quad \epsilon \longrightarrow \epsilon^{(1)}=\epsilon-2 \lambda \Delta \ln \phi
$$

where

$$
\theta[\psi, \phi]=\int_{\Gamma} d x_{\mu} \varepsilon_{\mu \nu}\left(\phi \partial_{\nu} \psi-\psi \partial_{\nu} \phi\right)
$$

with the following tensor notations: $\mu \in\{1,2\}, x_{\mu} \in\{x, y\}, \partial_{\mu}=\partial / \partial x_{\mu}, \varepsilon_{\mu \nu}$ is a fully antisymmetric tensor with $\varepsilon_{12}=1$, and summation is implied over repeated indices.

Proof. The proof is a direct computation. Choose the function $\phi$ as a prop solution. Note that a one-form, which is being integrated in the formula (6.3) is closed in the case when $\psi$ and $\phi$ are solutions of (6.2). Hence, the shape of the contour of integration $\Gamma$ in (6.3) is irrelevant. 
Then one can verify by direct substitution of (6.2) and (6.3) into the formulae (6.4) below that the dressed function $\psi^{(1)}$ satisfies the dressed equation (6.1) (with the potential $\epsilon^{(1)}(x, y)$ and the same spectral parameter value $\lambda$ ).

The Moutard transformation (6.2) can be iterated several times and the result can be expressed via Pfaffian forms [11]. Instead, we direct our interest to the Maxwell equations (1.2). A straightforward computation (recall, $\lambda=-c^{2} / \omega^{2}$ ) yields the expressions for the dressed electric and magnetic fields $\mathbf{E}^{(1)}$ and $\mathbf{B}^{(1)}$ :

$$
\begin{aligned}
\mathbf{E}^{(1)} & =e^{i \omega t}\left(0,0, \psi^{(1)}\right), \\
\mathbf{B}^{(1)} & =\frac{c}{\omega} e^{i \omega t}\left(-\psi_{y}^{(1)}, \psi_{x}^{(1)}, 0\right), \\
\mathbf{D}^{(1)} & =\epsilon^{(1)} \mathbf{E}^{(1)} .
\end{aligned}
$$

On the basis of (6.4), one can build a variety of exact solutions of the Maxwell equations.

EXAMPLE 6.2 (singular potentials). As a simple example, we dress $\epsilon=0$. This is not quite a medium, but one can easily proceed with formal calculations (6.2), (6.3), and (6.4) which result in a new "medium" whose dielectric permitivity $\epsilon^{(1)}(x, y)$ and the stationary component of the field $\psi^{(1)}$ are as follows:

$$
\begin{aligned}
\epsilon^{(1)} & =-\frac{8 c^{2}}{\omega^{2}} \frac{a^{\prime}(z) b^{\prime}(\bar{z})}{(a(z)+b(\bar{z}))^{2}}, \\
\psi^{(1)} & =\frac{a(z) \beta(\bar{z})-\alpha(z) b(\bar{z})+\xi(z, \bar{z})}{a(z)+b(\bar{z})},
\end{aligned}
$$

where

$$
\xi(z, \bar{z})=\int d z\left(\alpha(z) a^{\prime}(z)-a(z) \alpha^{\prime}(z)\right)+\int d \bar{z}\left(\beta^{\prime}(\bar{z}) b(\bar{z})-b^{\prime}(\bar{z}) \beta(\bar{z})\right),
$$

$a(z), \alpha(z), b(\bar{z})$, and $\beta(\bar{z})$ are arbitrary functions of $z=x+i y, \bar{z}=x-i y$. Note that the function $\psi^{(1)}$ from (6.2) and (6.4) provides in fact a general solution of the dressed equation, for it is described in terms of two arbitrary functions $\alpha(z)$ and $\beta(\bar{z})$. To ensure that the quantities found correspond to a physical nonabsorbing medium, one should require that the dressed dielectric permitivity function $\epsilon^{(1)}$ be real. This imposes an extra restriction to the quantities $a(z)$ and $b(\bar{z})$, namely, $b(\bar{z})=\overline{a(z)}$. Generally speaking, the functions $\epsilon^{(1)}$ and $\psi^{(1)}$ from (6.5) will have singularities along certain curves in the $(x, y)$-plane.

The reflexionless B-potentials for the one-dimensional problem (1.1) (see Example 3.1) possess point singularities on the real line (corresponding to zeroes of the function $u(x)$ ). Clearly, their 2D-analogues, such as (6.5) for (1.5), 
allow a much more diverse structure of singularities on the real plane. On the other hand, not requiring that the quantity $\epsilon^{(1)}$ be real, one obtains an absorbing medium which may not be devoid of interest for physical applications.

In conclusion, we study a dressing chain generated by the Moutard transformations (6.2). A simple periodic closing of the dressing chain results in a regular dielectric permitivity similar to the $1 \mathrm{D}$ case studied above.

Denote $f_{n}=\ln \phi$ and $f_{n+1}=\ln \psi^{(1)}$. Then, after a straightforward computation,

$$
\Delta\left(f_{n}+f_{n+1}\right)=\left\|\nabla f_{n}\right\|^{2}-\left\|\nabla f_{n+1}\right\|^{2}
$$

where $\|\cdot\|$ is the Euclidean norm.

The chain (6.7) is closely related to that of Veselov and Shabat [16] for the Schrödinger equation. Choosing $f_{n}$ specifically as

$$
f_{n}=\sqrt{\lambda_{n}} y+\int d x g_{n}(x),
$$

and substituting it into (6.7) (with $\lambda_{n}$ being constant), we obtain for the quantities $g_{n}(x)$ the following expression:

$$
\left(g_{n}+g_{n+1}\right)^{\prime}=g_{n}^{2}-g_{n+1}^{2}+\lambda_{n}-\lambda_{n+1},
$$

matching the corresponding formula of [16].

EXAMPLE 6.3 (regular dielectric permitivity). The simplest periodic closing of the dressing chain (6.7) is $f_{n+1}=f_{n}=F(x, y)$, which implies that the latter function $F$ is harmonic, and that the regular dielectric permitivity function in the corresponding medium is given by the formula

$$
\epsilon(x, y)=\frac{c^{2}}{\omega^{2}}\left(F_{x}^{2}+F_{y}^{2}\right)
$$

ACKNOWLEDGments. We are grateful to F. Gesztesy and two anonymous referees for a number of critical remarks and suggestions, which we believe have helped to improve both the conceptual and expository sides of this paper. This work was supported by RFBR Grant 00-01-00783, the Education Department of the Russian Federation Grant no. E00-3.1-383, and the National Science Foundation (NSF) Grant DMS 0072153.

\section{REFERENCES}

[1] A. B. Borisov and S. A. Zykov, The dressing chain of discrete symmetries and proliferation of nonlinear equations, Theoret. and Math. Phys. 115 (1998), 530-541.

[2] F. Calogero and A. Degasperis, Spectral Transform and Solitons. Vol. I, Studies in Mathematics and Its Applications, vol. 13, North-Holland Publishing, Amsterdam, 1982. 
[3] M. M. Crum, Associated Sturm-Liouville systems, Quart. J. Math. Oxford Ser. (2) 6 (1955), no. 2, 121-127.

[4] G. Darboux, Sur une proposition relative aux équations linéaries, C. R. Acad. Sci. Paris 94 (1882), 1456-1459 (French).

[5] L. A. Dmitrieva, Finite-gap solutions of the Harry Dym equation, Phys. Lett. A 182 (1993), no. 1, 65-70.

[6] - The higher-times approach to multisoliton solutions of the Harry Dym equation, J. Phys. A 26 (1993), no. 21, 6005-6020.

[7] F. Gesztesy and G. Teschl, On the double commutation method, Proc. Amer. Math. Soc. 124 (1996), no. 6, 1831-1840.

[8] W. Hereman, P. P. Banerjee, and M. R. Chatterjee, Derivation and implicit solution of the Harry Dym equation and its connections with the Korteweg-de Vries equation, J. Phys. A 22 (1989), no. 3, 241-255.

[9] C. G. J. Jacobi, Zur Theorie der Variationsrechnung und der Differentialgleichungen, J. reine angew. Math. 17 (1837), 68-82 (German).

[10] B. G. Konopel'chenko and V. G. Dubrovsky, Inverse spectral transform for the modified Kadomtsev-Petviashvili equation, Stud. Appl. Math. 86 (1992), no. 3, 219-268.

[11] V. B. Matveev and M. A. Salle, Darboux Transformations and Solitons, Springer Series in Nonlinear Dynamics, Springer-Verlag, Berlin, 1991.

[12] A. V. Mikhailov, A. B. Shabat, and R. I. Yamilov, The symmetry approach to classification of nonlinear equations. Complete lists of integrable systems, Russian Math. Surveys 42 (1987), no. 4, 1-63.

[13] V.S. Novikov, Reflexionless potentials of the acoustic problem, JETP Lett. 72 (2000), 223-234 (Russian).

[14] A. B. Shabat, Third version of the dressing method, Teoret. Mat. Fiz. 121 (1999), no. 1, 165-176 (Russian).

[15] S. I. Svinolupov and R. I. Yamilov, Explicit self-transformations for multifield Schrödinger equations and Jordan generalizations of the Toda chain, Teoret. Mat. Fiz. 98 (1994), no. 2, 207-219 (Russian).

[16] A. P. Veselov and A. B. Shabat, A dressing chain and the spectral theory of the Schrödinger operator, Funct. Anal. Appl. 27 (1993), no. 2, 81-96.

[17] A. V. Yurov, Darboux transformation for Dirac equations with $(1+1)$ potentials, Phys. Lett. A 225 (1997), no. 1-3, 51-59.

[18] Conjugate chains of discrete symmetries of $(1+2)$ nonlinear equations, Theoret. and Math. Phys. 119 (1999), no. 3, 731-738.

A. A. Yurova: Department of Mathematics, Kaliningrad State Technical University, Sovetsky Prospect 1, Kaliningrad 236000, Russia

E-mail address: yurova@bk.ru

A. V. Yurov: Department of Theoretical Physics, Kaliningrad State University, 14 Aleksandra Nevskogo Street, Kaliningrad 236041, Russia

E-mail address: artyom_yurov@mai 1 .ru

M. Rudnev: School of Mathematics, University of Bristol, University Walk, Bristol BS8 1TW, UK

E-mail address: M. Rudnev@bris.ac.uk 


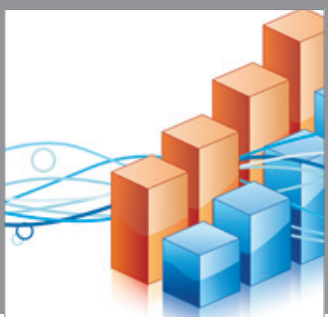

Advances in

Operations Research

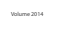

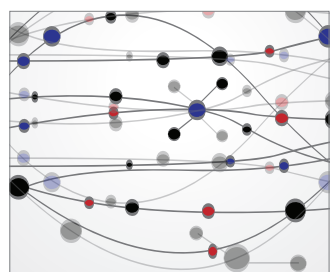

\section{The Scientific} World Journal
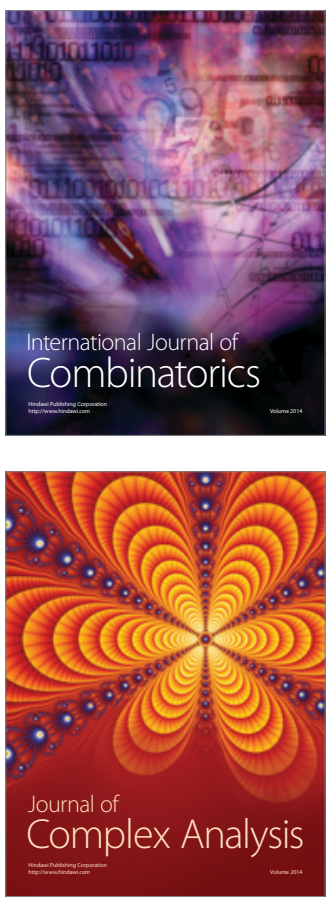

International Journal of

Mathematics and

Mathematical

Sciences
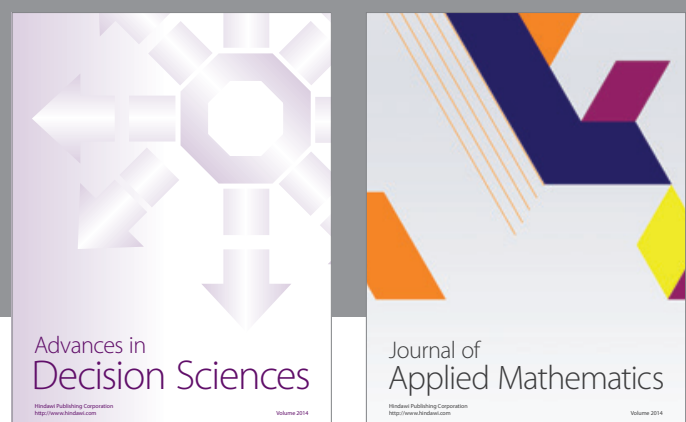

Journal of

Applied Mathematics
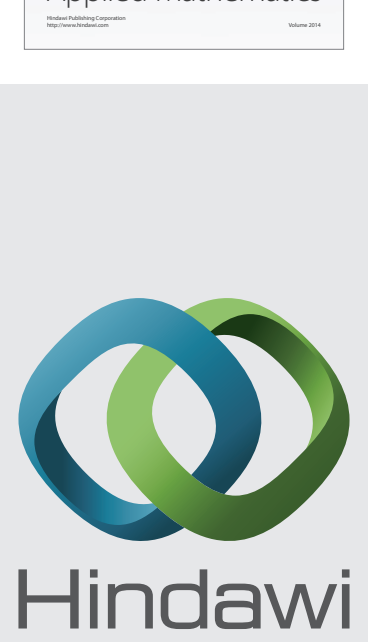

Submit your manuscripts at http://www.hindawi.com
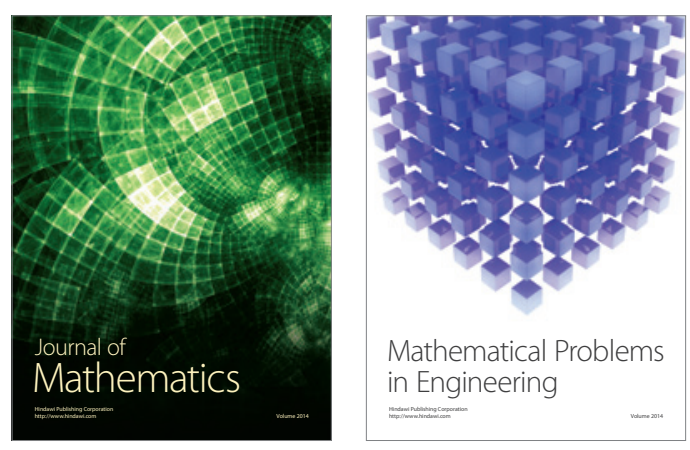

Mathematical Problems in Engineering
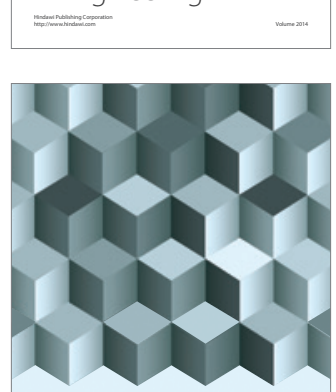

Journal of

Function Spaces
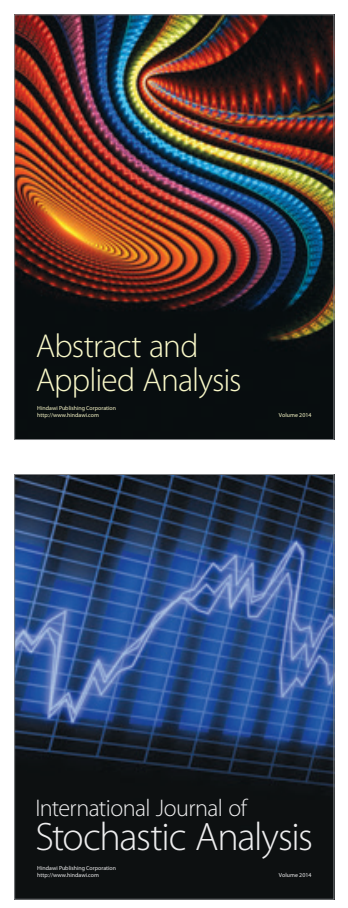

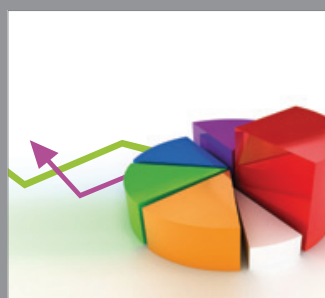

ournal of

Probability and Statistics

Promensencen
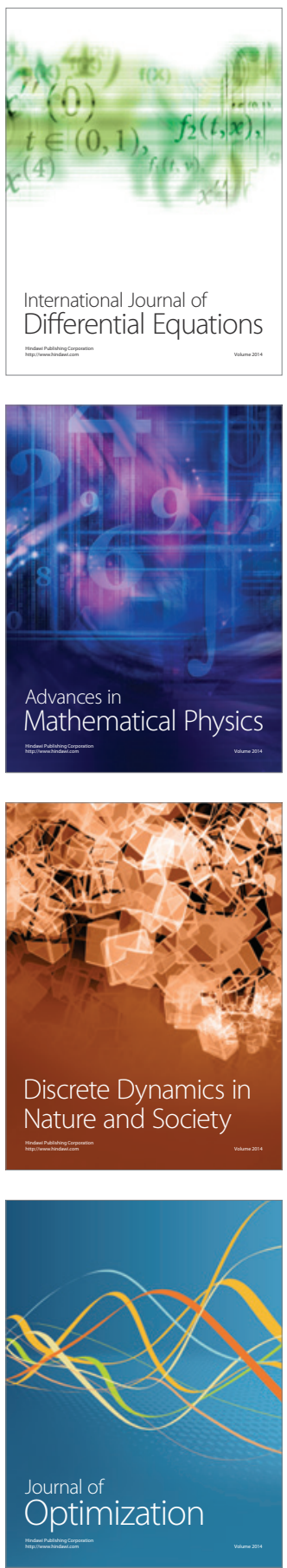